\title{
Irreversible Thermodynamic Discussions about Ferroelectric Phase Transitions
}

\author{
Jin Song Wang \\ Lin Yi University Network Center \\ Lin Yi Shandong china \\ Email: jsw1818@163.com
}

\begin{abstract}
The irreversibility of ferroelectric phase transitions has been studied by using the irreversible thermodynamics. The 'thermal hysteresis' of first-order ferroelectric phase transitions and the polydomain structure of ferroelectrics can be explained on the basis of the principle of minimum entropy production. A conclusion has been derived that the thermal hysteresis is not an intrinsic property of a system in which a first-order ferroelectric phase transition occurs. The finiteness of the system's surface is connected with the thermal hysteresis.
\end{abstract}

Keywords-Irreversible thermodynamics, ferroelectric phase transition, thermal hysteresis.

\section{INTRODUCTION}

Probing the complexity of matter world is an important job for people working at physics. Usually, the irreversible thermodynamics, the non-equilibrium statistical mechanics and the nonlinear dynamics are three tools for studying it, which are used from different angle. In particular, the interface (phase boundary) dynamics of first-order ferroelectric phase transitions has been the object of extensive experimental and theoretical studies.[1-11] And, the dynamic studies of domains and domain wall movements in ferroelectrics have made progress recently.[12,13] The topic of current interest is important as a means of probing multi-stable dynamical systems and also as a challenging example of nonlinear behavior. The irreversible thermodynamics for ferroelectric phase transitions has been just put forward.[11] However, they combine together and are hopeful to produce abundant results. Here, what we will do only is that we consider the irreversibility of ferroelectric phase transitions, which is not considered in the equilibrium

thermodynamics, and, make some irreversible thermodynamic discussions about the 'thermal hysteresis' [14] and the emergence of polydomain structure in the process of a ferroelectric phase transition so as to establish a firm base for these irreversible phenomena.

The thermal hysteresis of a first-order ferroelectric phase transition is an irreversible phenomenon obviously. But it was treated by using the equilibrium thermodynamics for ferroelectric phase transitions, the well-known LandauGinzburg-Devonshire theory.[14] So, there is an inherent contradiction in this case. Let it be. In the Landau-GinzburgDevonshire theory, the thermal hysteresis is thought to be due to a series of metastable states existing around the Curie temperature (phase transition temperature) $T_{C}$. In fact, these metastable states and all equilibrium states can be regarded as the so-called stationary states in irreversible thermodynamics. When a system reaches a stationary state, the local entropy production is a minimum - the so-called principle of minimum entropy production.[15] The ferroelectric phase transition may be regarded as a stationary state process.

In Ref.[11], we derived the rate of local entropy production $\sigma_{s}$ from the Gibbs equation

$$
T d s=d u-\vec{E} \cdot d \vec{D}-\sum_{i} \mu_{i} d n_{i}
$$

where $T, \vec{E}, \vec{D}$ is the temperature, the electric field intensity and the electric displacement within an arbitrary small volume, respectively; $s, u, \mu_{i}, n_{i}$ is the entropy density, the internal energy density, the chemical potential and the molar quantity density in the small volume, respectively. Differentiating Eq.(1), we had

$$
\begin{aligned}
& \frac{\partial s}{\partial t}=\frac{1}{T}\left[\frac{\partial u}{\partial t}-\vec{E} \cdot \frac{\partial \vec{D}}{\partial t}-\sum_{i} \mu_{i} \frac{\partial n_{i}}{\partial t}\right] \\
& =-\frac{1}{T} \vec{\nabla} \cdot \vec{J}_{u}-\frac{\vec{E}}{T} \cdot \vec{J}_{P}+\sum_{i} \frac{\mu_{i}}{T} \vec{\nabla} \cdot \vec{J}_{n i}
\end{aligned}
$$$$
\text { where } \vec{J}_{u}, \vec{J}_{P}, \vec{J}_{n i} \text { is the energy flux, the polarization }
$$
current and the matter flux, respectively. Considering $\vec{J}_{u}$ should consist of three parts

$$
\vec{J}_{u}=\vec{J}_{q}+\phi \vec{J}_{P}+\sum_{i} \mu_{i} \vec{J}_{n i}
$$

where $\vec{J}_{q}$ is the heat flux, $\phi$ is the electrical potential and satisfies

$$
\vec{E}=-\vec{\nabla} \phi
$$

we deduced Eq.(2) and obtained the local entropy balance equation

$$
\frac{\partial s}{\partial t}+\vec{\nabla} \cdot \vec{J}_{s}=\sigma_{s}
$$

where the entropy flux and the rate of local entropy production are

$$
\vec{J}_{s}=\frac{\vec{J}_{q}+\phi \vec{J}_{P}}{T}
$$




$$
\sigma_{s}=\vec{J}_{q} \cdot \vec{\nabla}\left(\frac{1}{T}\right)+\vec{J}_{P} \cdot \vec{\nabla}\left(\frac{\phi}{T}\right)-\sum_{i} \frac{\vec{J}_{n i}}{T} \cdot \vec{\nabla} \mu_{i}
$$

respectively. Because the system is in the crystalline states before and after a phase transition then there is no diffusion of any one kind of particles in the system, $\vec{J}_{n i}=0$. The rate of local entropy production can be reduced as

$$
\sigma_{s}=\vec{J}_{q} \cdot \vec{\nabla}\left(\frac{1}{T}\right)+\vec{J}_{P} \cdot \vec{\nabla}\left(\frac{\phi}{T}\right)
$$

Then we took the inner electric field into account and obtained the rate of local entropy production in the case of not applying the outer electric field

$$
\sigma_{s}=\vec{J}_{q} \cdot \vec{\nabla}\left(\frac{1}{T}\right)+\vec{J}_{P} \cdot \vec{\nabla}\left(\frac{\phi_{i}}{T}\right)
$$

where $\phi_{i}$ is the inner electrical potential.

The inner electrical potential $\phi_{i}$, generally speaking, depends on the factors such as the temperature, the stress, etc. In our problem under discussion, the temperature is the main factor. As a simplified assumption, $\phi_{i}$ is a function of the temperature only:

$$
\phi_{i}=\left\{\begin{array}{l}
\phi_{i}(T), T<T_{C} \\
0, T>T_{C}
\end{array}\right.
$$

Eq.(9) can be re-written as

$$
\sigma_{s}=-\left[\vec{J}_{q}+\vec{J}_{P}\left(\phi_{i}-T \frac{\partial \phi_{i}}{\partial T}\right)\right] \frac{\vec{\nabla} T}{T^{2}}
$$

A system where a first-order ferroelectric phase transition occurs is heterogeneous. The situations differ radically among the region where the phase transition has occurred, the region where the phase transition is occurring, the region where the phase transition will occur.[11] In the region where the phase transition is occurring, the flux $\vec{J}_{q}=0$

for the pure heat conduction and the heat conduction induced by the thermal-electric coupling cancel out each other so as to release or absorb the latent heat; the other flux $\vec{J}_{P}=\frac{\partial \vec{D}}{\partial t}=\frac{\partial \vec{D}}{\partial T} \frac{\partial T}{\partial t}$ tends to infinity. No doubt that the principle of minimum entropy production prohibits any one stationary state from owning infinite magnitude of $\sigma_{s}$. According to Eq.(11), $\vec{\nabla} T$ should tend to zero in this region. This means, the temperature of outer side of this region tends to unchanged but that of inner side of this region tends to change more quickly so as to reduce $\vec{\nabla} T$ (It is impossible for the temperature of outer side of this region to change in the opposite direction). These factors make this region absorb (or release) more heat from (or to) the outer, adjacent region where the phase transition has occurred. This lead to the latent heat and is consistent with $\vec{J}_{q}=0$. The phase transition occurs firstly in the surface layer of the system and moves unceasingly into the inner part of it. A constant temperature gradient is kept in the outer region where the phase transition has occurred.[11] The temperature of it, which is usually regarded as the temperature of the whole system in experiments, must higher (or lower) than the Curie temperature-this may lead to the thermal hysteresis.

No doubt that the shape and the area of surface can greatly affect the above process. We can conclude that the thermal hysteresis can be reduced if the system has a larger specific surface and, the thermal hysteresis can be neglected if a finite system has an extremely-large specific surface. So, the thermal hysteresis is not an intrinsic property of the system. We suggest strongly that experimentalists should make some efforts to test it.

The region where the phase transition will occur can be regarded as an equilibrium system for there is no restriction on the two forces $\vec{X}_{P} \quad\left(=\vec{\nabla}\left(\frac{\phi_{i}}{T}\right) \quad\right.$ and $\vec{X}_{q}\left(=\vec{\nabla}\left(\frac{1}{T}\right)\right.$ ).[11] In other words, the forces and the corresponding fluxes are zero in this region. To a system where a second-order ferroelectric phase transition occurs, the case is just like that of the region where a first-order ferroelectric phase transition will occur. The spontaneous polarization is continuous at the Curie temperature $T_{C}$ and change from zero to a infinitesimal magnitude (in the processes of heating) or conversely (in the processes of cooling). This means $\vec{X}_{q}, \vec{X}_{P}$ and $\vec{J}_{q}, \vec{J}_{P}$ can be arbitrary infinitesimal magnitudes. The second-order phase transition occurs in every part of the system simultaneously, i.e., there is no the co-existence of two phases. So, there is no the latent heat and the thermal hysteresis.

For a ferroelectric phase transition can be considered as a stationary state process, the rate of local entropy production should be a positive minimum. Usually, there are several possible orientations of the spontaneous polarization $\vec{P}$ in the system. According to Eq.(11) and $\vec{J}_{q}=0$ in

the region where the phase transition is occurring, we may conclude that when the system changes from the paraelectric phase to the ferroelectric phase the orientation of $\vec{P}$ must take the best one so as to satisfy the principle of minimum entropy production (because $\vec{J}_{P}=\frac{\partial \vec{D}}{\partial t}=\frac{\partial\left(\varepsilon_{0} \vec{E}_{i}+\vec{P}\right)}{\partial t}, \vec{E}_{i}$ is the intensity of the inner electric field). It is noted that the variation of direction of $\vec{\nabla} T$ in the system is continuous for the system is finite (i.e., existence of surface). So, $\vec{P}$ at different location generally has different orientation. The ferroelectric polydomain structure thus emerges. It should be emphasized that the 
point of view from which we obtain the conclusion is different from that in Ref.[16], which is based on the principle of free energy minimization. The difference is that we made a dynamical description but others proved the rationality of existence of polydomain structure only.

To a second-order ferroelectric phase transition, a system where it occurs is in equilibrium; and, to a first-order ferroelectric phase transition, in another system the region where it will occur is in equilibrium. So, the phase transitions are equilibrium not non-equilibrium ones. The polydomain structure should be a kind of equilibrium not dissipative one. The latter is far from equilibrium and is also called the selforganized structure.[17]

We regarded the inner electrical potential $\phi_{i}$ as a function of the temperature only. Thus, $\vec{X}_{q}$ will not be independent of $\vec{X}_{P}$

$$
\left[T \frac{d \phi_{i}}{d T}-\phi_{i}(T)\right] \vec{X}_{q}(T)+\vec{X}_{P}(T)=0
$$

this leads to the conditions that the rate of local entropy production is a minimum (see Eq.(30) and (31) in Ref.[11])

$$
\left(\frac{\partial \sigma_{s}}{\partial \vec{X}_{q}}\right)_{\vec{X}_{P}}=2 L_{q q} \vec{X}_{q}+2 L_{q P} \vec{X}_{P}=2 \vec{J}_{q}=0
$$

$$
\left(\frac{\partial \sigma_{s}}{\partial \vec{X}_{P}}\right)_{\vec{X}_{q}}=2 L_{P q} \vec{X}_{q}+2 L_{P P} \vec{X}_{P}=2 \vec{J}_{P}=0
$$

become invalid. So, $\phi_{i}$ must not be a function of temperature only. It should be related to the factor of stress, etc. Thus, a ferroelectric phase transition must have the effect of deformation of the system - this is a necessary demand of the irreversible thermodynamics and is consistent with the facts. Another factor that leads to the thermal hysteresis may be the stress. To a first-order ferroelectric phase transition, there is always a sudden change of the system's volume and shape. The inner part of it has to overcome the bound of the outer part, where the phase transition occurs earlier. So, the transition-occurrence in the inner part may be delayed. For the stress is a second-order tensor and makes the problem more complex, we will deal with it in another paper.

\section{REFERENCES}

[1] A. Gordon, Phys. Lett. A 99 (1983) 329.

[2] J. Dec, Ferroelectrics 69 (1986) 181.

[3] A. Gordon, Physica B 138 (1986) 239.

[4] J. Dec, J. Phys. C 21 (1988) 1257.

[5] A. Gordon, Phys. Lett. A 154 (1991) 79.

[6] J. A. Tuszynskii and D. Sept, J. Phys. Cond. Matter 6 (1994) 3583. 